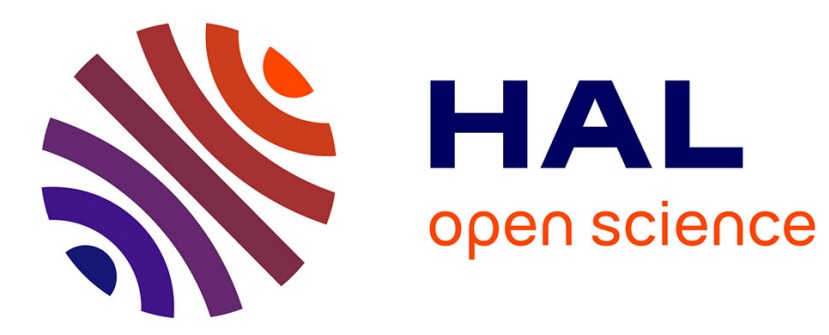

\title{
Hamilton Cycle Decomposition of the Butterfly Network
}

Jean-Claude Bermond, Eric Darrot, Olivier Delmas, Stéphane Pérennes

\section{To cite this version:}

Jean-Claude Bermond, Eric Darrot, Olivier Delmas, Stéphane Pérennes. Hamilton Cycle Decomposition of the Butterfly Network. RR-2920, INRIA. 1996. inria-00073777

\section{HAL Id: inria-00073777 \\ https://hal.inria.fr/inria-00073777}

Submitted on 24 May 2006

HAL is a multi-disciplinary open access archive for the deposit and dissemination of scientific research documents, whether they are published or not. The documents may come from teaching and research institutions in France or abroad, or from public or private research centers.
L'archive ouverte pluridisciplinaire HAL, est destinée au dépôt et à la diffusion de documents scientifiques de niveau recherche, publiés ou non, émanant des établissements d'enseignement et de recherche français ou étrangers, des laboratoires publics ou privés. 


\section{Hamilton cycle decomposition of the Butterfly network}

Jean-Claude Bermond, Eric Darrot, Olivier Delmas, Stéphane Perennes

$\mathbf{N}^{\circ} 2920$

Juin 1996

THÈME 1 



\title{
RI N R I I A
}

\section{Hamilton cycle decomposition of the Butterfly network}

\author{
Jean-Claude Bermond, Eric Darrot, Olivier Delmas, Stéphane Perennes \\ Thème 1 - Réseaux et systèmes \\ Projet SLOOP
}

Rapport de recherche ${ }^{\circ} 2920$ - Juin 1996 - 16 pages

Abstract: in this paper, we prove that the wrapped Butterfly graph $\mathcal{W B F}(d, n)$ of degree $d$ and dimension $n$ is decomposable into Hamilton cycles. This answers a conjecture of D. Barth and A. Raspaud who solved the case $d=2$.

Key-words: Butterfly graph, graph theory, Hamiltonism, Hamilton decomposition, Hamilton cycle, Hamilton circuit, perfect matching.

This work has been supported by the CEFIPRA (French-Indian collaboration) and the European project HCM MAP.

Version of June 28, 1996 to appear in PARALlel Processing Letters.

Email: \{bermond, darrot, delmas, sp\}@unice.fr 


\section{Décomposition en cycles Hamiltoniens du réseau Butterfly}

Résumé : dans cet article, nous prouvons que le graphe Butterfly rebouclé $\mathcal{W B F}(d, n)$ de degré $d$ et de dimension $n$ est décomposable en cycles Hamiltoniens. Ce résultat répond à une conjecture de D. Barth et A. Raspaud qui ont résolu le cas $d=2$.

Mots-clé : graphe Butterfly, théorie des graphes, Hamiltonisme, décomposition Hamiltonienne, cycle Hamiltonien, circuit Hamiltonien, couplage parfait. 


\section{Introduction and notations}

The construction of one, and if possible many edge-disjoint Hamilton cycles in a network can provide advantage for algorithms that make use of a ring structure. As example, the existence of many edge-disjoint Hamilton cycles allows the message traffic to be evenly distributed across the network. Furthermore, a partition of the edges into Hamilton cycles can be used in various distributed algorithms (termination, garbage collector,...). So, many authors have considered the problem of finding how many edge-disjoint Hamilton cycles can be found in a given network. The most significant results have been obtained for the class of Cayley graphs on abelian groups, and for (underlying) line digraphs. Here we solve this problem for the Butterfly networks. These networks have been proposed as suitable topologies for parallel computers, due to their interesting structure (see $[8,9])$ because they are, when properly defined, both Cayley digraphs (on a non-abelian group) and iterated line digraphs.

\subsection{Definitions}

First, we have to warn the reader that under the name Butterfly and with the same notation, different networks are described. Indeed, if some authors consider the Butterfly network as a multistage network used to route permutations, others consider it as point-to-point network. In what follows, we will study the point-to-point version, and use Leigthon's terminology [8], namely, wrapped Butterfly. Also, when we use the terms edge-disjoint or arc-disjoint, it obviously means pairwise edge-disjoint or arc-disjoint. In this article, we will use the next definitions and notations. For definitions not given here, see [9].

$\mathbb{Z}_{q}$ will denote the set of integers modulo $q$; addition of elements in $\mathbb{Z}_{q}$ will always occur in $\mathbb{Z}_{q}$.

Definition 1.1 The wrapped Butterfly digraph of degree $d$ and dimension $n$, denoted $\mathcal{W B} \mathcal{B}(d, n)$, has as vertices the couples $(x, l)$ where $x$ is an element of $\mathbb{Z}_{d}^{n}$, that is, a word $x_{n-1} x_{n-2} \cdots x_{1} x_{0}$ where the letters belong to $\mathbb{Z}_{d}$, and $l \in \mathbb{Z}_{n}$ ( $l$ is called the level). For any $l$, a vertex $\left(x_{n-1} x_{n-2} \cdots\right.$ $\left.x_{l} \cdots x_{1} x_{0}, l\right)$ is joined by an arc to $d$ vertices $\left(x_{n-1} \cdots x_{l+1}, x_{l}+\alpha, x_{l-1} \cdots x_{0}, l+1\right)$ where $\alpha$ is any element of $\mathbb{Z}_{d}$. Each one of these arcs is said to have the slope $\alpha$.

$\mathcal{W} \overrightarrow{\mathcal{B}} \mathcal{F}(d, n)$ is a $d$-regular digraph with $n d^{n}$ vertices; its diameter is $2 n-1$. This network is sometimes considered as undirected, but its structure being indeed directed, we will always consider the digraph.

For convenience, we repeat the level 0 when drawing the wrapped Butterfly digraph. Hence, the reader has to remember that the two occurrences of level 0 have to be identified. Figure (1) displays $\mathcal{W B \mathcal { B }} \mathcal{F}(3,2)$ with the arcs directed from left to right. Note that $\mathcal{W B} \mathcal{B}(d, n)$ is often represented (for example in $[8,9]$ ) in an opposite way to our drawing as the authors denote the nodes $\left(x_{0} x_{1} \cdots x_{n-1}, l\right)$. 


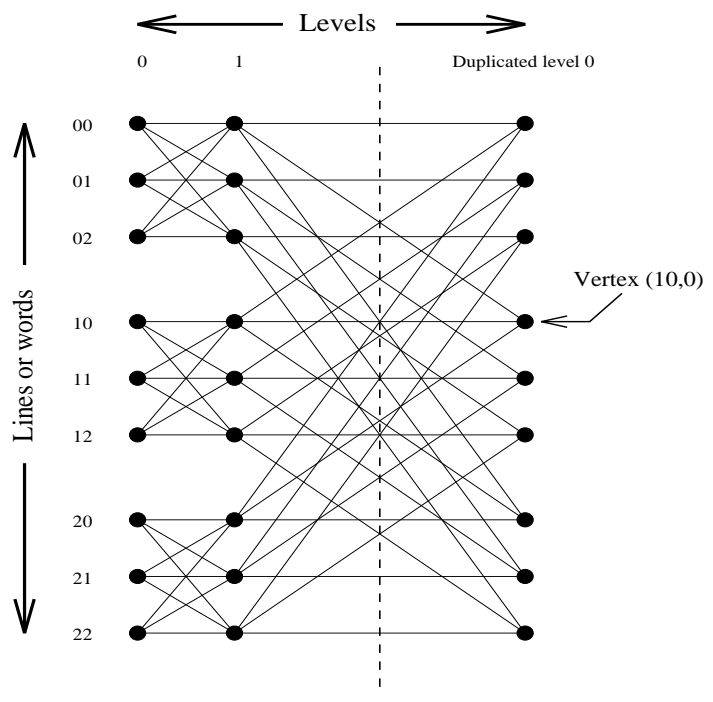

Figure 1: The digraph $\mathcal{W} \overrightarrow{\mathcal{B}} \mathcal{F}(3,2)$, the arcs beings directed from left to right.

Now, we give two digraph definitions we use in the following.

- $\mathcal{K}_{d}^{+}$will denote the complete symmetric digraph with a loop on each vertex,

- $\overrightarrow{\mathcal{K}}_{d, d}$ will denote the complete bipartite digraph where each set of the bipartition has size $d$ and with all the arcs directed from the left part to the right part.

Note that $\mathcal{W} \overrightarrow{\mathcal{B}} \mathcal{F}(d, 1)$ is nothing else than $\mathcal{K}_{d}^{+}$.

In digraphs, the concept of dipaths and circuits (directed cycles) is well-known. Here, we need to use more general concepts valid for digraphs of paths and cycles (which are also called oriented elementary paths and oriented elementary cycles).

Definition 1.2 A path of a digraph is a sequence $\mu=\left(v_{0}, e_{0}, v_{1}, e_{1}, \cdots, v_{k}, e_{k}, v_{k+1}\right)$ where the $v_{i}$ 's are vertices and the $e_{i}$ 's are arcs such that the end vertices of $e_{i}$ are $v_{i}$ and $v_{i+1}$ and where the sequence $\mu$ does not meet twice the same vertex except maybe $v_{0}$ and $v_{k+1}$.

Definition 1.3 A path such that $v_{k+1}=v_{0}$ in the sequence $\mu$ is called a cycle.

Note that the arc $e_{i}$ can be either directed from $v_{i}$ to $v_{i+1}$ or from $v_{i+1}$ to $v_{i}$. If all the arcs of the path (resp. cycle) are directed from $v_{i}$ to $v_{i+1}$ we have a dipath (resp. circuit also called dicycle).

Definition 1.4 A vertex $v_{i}$ of a cycle is said to be of type + (resp. of type -) for the cycle, if $v_{i}$ is the terminal vertex of $e_{i-1}$ (resp. $e_{i}$ ) and the initial vertex of $e_{i}$ (resp. $e_{i-1}$ ). 
Note that in a circuit, all vertices are of type + , but the type is not necessarily defined for all the vertices of a cycle.

Definition $1.5 A$ vertex $v$ is said to be crossed by a cycle, or a cycle crosses the vertex $v$, if $v$ is of type + or of type - for the cycle. When a vertex $v$ is crossed by a cycle, we will define its sign function $\epsilon$ by $\epsilon(v)=+1$ (resp. $\epsilon(v)=-1$ ) if $v$ is of type + (resp. of type -).

Remark 1 We can also define the predecessor $p(v)$ and the successor $s(v)$ of the vertex $v$ in the order induced by the cycle. Then, the vertex $v$ is of type + (or has sign $\epsilon(v)=+1)$ if $(p(v), v)$ and $(v, s(v)$ ) are both arcs of the digraph, and is of type - (or has sign $\epsilon(v)=-1)$ if both $(s(v), v)$ and $(v, p(v))$ are arcs of the digraph.

Definition 1.6 A Hamilton cycle (resp. circuit) of a digraph is a cycle (resp. circuit) which contains every vertex exactly once.

Definition 1.7 We will say that a digraph is decomposable into Hamilton cycles (resp. circuits) if its arcs can be partitioned into Hamilton cycles (resp. circuits).

Definition 1.8 A Hamilton cycle of $\mathcal{W} \overrightarrow{\mathcal{B}} \mathcal{F}(d, n)$ will be said to be $l$-crossing if the cycle crosses all the vertices of level $l$ and furthermore $\sum_{v=(x, l), x \in \mathbb{Z}_{d}^{n}} \epsilon(v) \equiv 0 \quad(\bmod d)$.

Figure (3) shows examples of 1-crossing Hamilton cycles in $\mathcal{W B \mathcal { B }} \mathcal{F}(3,2)$ and $\mathcal{W B} \mathcal{B}(3,3)$.

\subsection{Results}

Various results have been obtained on the existence of Hamilton cycles in classical networks (see for example the surveys [2,7]). For example, it is well-known that any Cayley graph on an abelian group is hamiltonian. Furthermore, it has been conjectured by Alspach [1] that:

Conjecture 1 (Alspach) Every connected Cayley graph on an abelian group has a Hamilton decomposition.

This conjecture has been verified for all connected 4-regular graphs on abelian groups in [6]. It includes in particular the toroidal meshes (grids). For the hypercube, it is also known that $\mathcal{H}(2 d)$ is decomposable into $d$ Hamilton cycles (see [2,3]).

The wrapped Butterfly digraph is actually a Cayley graph (on a non-abelian group) and a line digraph. So, the decomposition into Hamilton cycles (resp. circuits) of this digraph has received some attention. It is well-known that $\mathcal{W B \mathcal { B }} \mathcal{F}(d, n)$ has one Hamilton circuit (see [8, page 465] for a proof in the case $d=2$ or [12]). In [4], Barth and Raspaud proved that the underlying multigraph associated to $\mathcal{W B} \mathcal{F}(2, n)$ contains two arc-disjoint Hamilton cycles answering a conjecture of J. Rowley and D. Sotteau [10]. In our terminology, their result can be stated as:

RR n 2920 
Theorem 1.1 (Barth, Raspaud) $\mathcal{W} \overrightarrow{\mathcal{B}} \mathcal{F}(2, n)$ is decomposable into Hamilton cycles.

They conjectured that this result can be generalized for any degree:

Conjecture 2 (Barth, Raspaud) For $n \geq 2, \mathcal{W B} \mathcal{B}(d, n)$ is decomposable into Hamilton cycles.

In this paper, we prove the conjecture (2). To do so, we use some techniques introduced in [5] where we studied the decomposition of $\mathcal{W B \mathcal { B }} \mathcal{F}(d, n)$ into Hamilton circuits. In fact, we prove that $\mathcal{W B} \mathcal{F}(d, n)$ is decomposable into $d \quad l$-crossing Hamilton cycles. Indeed, the $l$-crossing property, combined with the recursive structure of $\mathcal{W B} \mathcal{F}(d, n)$, enables us to prove that the number of $l$-crossing arc-disjoint Hamilton cycles that $\mathcal{W B} \mathcal{F}(d, n)$ contains can only increase when $n$ increases. Then, we prove mainly that $\mathcal{W B} \mathcal{F}(d, 2)$ contains $d$ arc-disjoint $l$-crossing Hamilton cycles, by constructing two arc-disjoint $l$-crossing Hamilton cycles using only arcs of slopes 0 and 1 and $d-2$ arcdisjoint Hamilton circuits using arcs of other slopes. The results are summarized in the following theorem:

Theorem 1.2 For $n \geq 2$,

- for $d \notin\{3,4,6\}, \mathcal{W B} \mathcal{B}(d, n)$ is decomposable into $d-2$ Hamilton circuits and 2 Hamilton cycles,

- for $d \in\{4,6\}, \mathcal{W B} \mathcal{B}(d, n)$ is decomposable into Hamilton circuits,

- $\mathcal{W B} \mathcal{B}(3, n)$ is decomposable into 1 Hamilton circuit and 2 Hamilton cycles.

\section{The general construction}

We give below some additional definitions and properties enabling us to establish lemma (2.2) which is indeed a strengthened version of the inductive lemma of [5]. This lemma is then applied in section (3) to construct inductively the decomposition.

\subsection{Families of perfect matchings}

We will denote by $M$ a permutation of $\mathbb{Z}_{d}$ mapping $a$ to $M(a)$ or equivalently the associated perfect matching of $\overrightarrow{\mathcal{K}}_{d, d}$ which contains all the arcs $(a, M(a))$.

Definition 2.1 Let $S$ be a set of slopes (that is a subset of $\mathbb{Z}_{d}$ ). Then, a perfect matching $M$ of $\overrightarrow{\mathcal{K}}_{d, d}$ uses the slopes in $\mathbf{S}$ if, for any $a \in \mathbb{Z}_{d} M(a) \in\{a+s, s \in S\}$. A family of perfect matchings $\mathcal{M}=\left\{M_{x}, x \in \mathbb{Z}_{d}^{n}\right\}$ of $\overrightarrow{\mathcal{K}}_{d, d}$ uses the slopes in $\mathbf{S}$ if, for any perfect matching $M_{x}$ of the family, $M_{x}$ uses the slopes in $S$.

Definition 2.2 For $1 \leq j \leq p$, let $\mathcal{M}_{j}=\left\{M_{x, j} \mid x \in \mathbb{Z}_{d}^{n}\right\}$ be $p$ families of perfect matchings. The families $\mathcal{M}_{j}$ are said to be compatible if, for each $x$ in $\mathbb{Z}_{d}^{n}$, the perfect matchings $M_{x, j}$ are arc-disjoint (i.e. $\forall a M_{x, j}(a) \neq M_{x, j^{\prime}}(a)$, for $\left.j \neq j^{\prime}\right)$. 
Definition 2.3 A family $\mathcal{M}=\left\{M_{x}, x \in \mathbb{Z}_{d}^{n}\right\}$ of $\overrightarrow{\mathcal{K}}_{d, d}$ perfect matchings satisfies the cyclic-potent property if, for any order of composition of the $M_{x}$ and any set of sign $\left\{\epsilon_{x} \mid x \in \mathbb{Z}_{d}^{n} \epsilon_{x} \in\{-1,1\}\right\}$ such that $\sum_{x} \epsilon_{x} \equiv 0 \bmod d$, the permutation $\Pi_{x} M_{x}^{\epsilon_{x}}$ is cyclic.

Definition 2.4 A family of perfect matchings $\mathcal{M}=\left\{M_{x}, x \in \mathbb{Z}_{d}^{n}\right\}$ is of type $(i, j)$ if:

- for $x \neq 0, M_{x}(a)=a+i$;

- for $x=0, M_{0}(a)=a+j$.

Lemma 2.1 A family of perfect matchings of type $(i, j) \mathcal{M}=\left\{M_{x}, x \in \mathbb{Z}_{d}^{n}\right\}$ is cyclic-potent if and only if $j-i$ is prime with $d$.

Proof. As the permutations of the family commute, the permutation $\Pi_{x} M_{x}^{\epsilon_{x}}$ of definition (2.3) can be simply expressed as $x \rightarrow x+\delta$. So, this permutation will be cyclic if and only if $\delta$ is prime with $d$. Here $\delta=\left(\sum_{x \neq 0} \epsilon_{x}\right) i+\epsilon_{0} j$. As $\sum_{x} \epsilon_{x}=0$, we have $\delta=\left(\sum_{x} \epsilon_{x}\right) i+\epsilon_{0}(j-i)=\epsilon_{0}(j-i)$. So, $\delta$ is clearly prime with $d$ if and only if $j-i$ is prime with $d$.

We will represent a set of $p$ families of perfect matchings of type $(i, j):\left\{\left(i_{0}, j_{0}\right),\left(i_{1}, j_{1}\right), \ldots\right.$, $\left.\left(i_{p-1}, j_{p-1}\right)\right\}$ by the array:

$$
\begin{array}{lllllll}
i_{0} & i_{1} & i_{2} & i_{3} & \ldots & i_{p-2} & i_{p-1} \\
j_{0} & j_{1} & j_{2} & j_{3} & \ldots & j_{p-2} & j_{p-1}
\end{array}
$$

In section (3) we will need some very simple cyclic-potent families of perfect matchings that we give as examples.

Families 1 There exist $d$ compatible cyclic-potent families of perfect matchings:

$$
\begin{array}{ccccccccc}
0 & 1 & 2 & 3 & 4 & 5 & \ldots & d-2 & d-1 \\
1 & 2 & 3 & 4 & 5 & 6 & \ldots & d-1 & 0
\end{array}
$$

These families are cyclic-potent as, applying lemma (2.1), $1-0=2-1=\cdots=d-1-(d-2)=$ $0-(d-1)=1$ which is prime with $d$. These families use all the slopes.

Families 2 There exist 2 compatible families which use the slopes $\{0,1\}$ :

$$
\begin{array}{ll}
0 & 1 \\
1 & 0
\end{array}
$$

According to lemma (2.1) they are two compatible cyclic-potent families and they use the slopes $\{0,1\}$.

RR n 2920 
Families 3 When $d \neq 3$, there exist $d-2$ compatible cyclic-potent families of perfect matchings using the slopes $\{2, \ldots, d-1\}$. One possible solution is given below:

- When $d$ is odd and $d \neq 3$, the following families can be used:

$\begin{array}{cccccccc}2 & 3 & 4 & 5 & \ldots & d-3 & d-2 & d-1 \\ 4 & 5 & 6 & 7 & \ldots & d-1 & 2 & 3\end{array}$

- When $d$ is even, we use the following families:

$$
\begin{array}{lllllll}
2 & 3 & 4 & 5 & \ldots & d-2 & d-1 \\
3 & 2 & 5 & 4 & \ldots & d-1 & d-2
\end{array}
$$

These families are cyclic-potent as, applying lemma (2.1), we get:

- for $d$ odd, $4-2=5-3=\cdots=d-1-(d-3)=2$ and $2-(d-2)=3-(d-1)=4$, as 2 and 4 are prime with $d$;

- for $d$ even, $3-2=5-4=\cdots=(d-1)-(d-2)=1$ and $2-3=4-5=\cdots$ $=(d-2)-(d-1)=-1$, which are prime with $d$.

In both cases, the slopes used are in $\{2, \ldots, d-1\}$.

\subsection{Inductive construction}

Lemma 2.2 If $\mathcal{W B} \mathcal{B}(d, n)$ admits $p$ arc-disjoint l-crossing Hamilton cycles and if there exist $p$ cyclic-potent families of perfect matchings in $\overrightarrow{\mathcal{K}}_{d, d}$, then $\mathcal{W B} \mathcal{B}(d, n+1)$ admits $p$ arc-disjoint l-crossing Hamilton cycles.

Proof. Let $H$ be an $l$-crossing Hamilton cycle of $\mathcal{W B \mathcal { B }} \mathcal{F}(d, n)$. As all the levels are equivalent, we can suppose without loss of generality and for simplicity in the notations that $l=0$. Let $\mathcal{M}=\left\{M_{x}, x \in \mathbb{Z}_{d}^{n}\right\}$ be a cyclic-potent family of perfect matchings of $\overrightarrow{\mathcal{K}}_{d, d}$. The vertices of $\mathcal{W} \overrightarrow{\mathcal{B}} \mathcal{F}(d, n+1)$ can be labeled $(a x, l)$ with $a \in \mathbb{Z}_{d}, x \in \mathbb{Z}_{d}^{n}$ and $l \in \mathbb{Z}_{n+1}$. Now, we associate to $H$ and $\mathcal{M}$ a partial digraph $H^{\prime}$ in $\mathcal{W} \overrightarrow{\mathcal{B}} \mathcal{F}(d, n+1)$ as follows (for an example of such a construction see figure (3)):

- for $0 \leq l \leq n-1$ and for each $a$, if the arc $(x, l)\left(x^{\prime}, l+1\right)$ belongs to $H$, we put in $H^{\prime}$ the arc $(a x, l)\left(a x^{\prime}, l+1\right)$ where the indices are taken modulo $n+1$, which means that to the arc $(x, n-1)\left(x^{\prime}, 0\right)$ of $H$ is associated the $\operatorname{arc}(a x, n-1)\left(a x^{\prime}, n\right)$ in $H^{\prime}$;

- between levels $n$ and 0 of $\mathcal{W} \overrightarrow{\mathcal{B}} \mathcal{F}(d, n+1)$ we put the arcs joining $(a x, n)$ to $\left(M_{x}(a) x, 0\right)$.

With such a definition, each vertex of $\mathcal{W B} \mathcal{F}(d, n+1)$ is incident to two arcs of $H^{\prime}$. Hence, we can define for each vertex a predecessor and a successor on $H^{\prime}$ that enables us to prove that we can order $H^{\prime}$ in a cycle. 
For $1 \leq l \leq n-1$, let $\left(x^{\prime}, l^{\prime}\right)$ (resp. $\left(x^{\prime \prime}, l^{\prime \prime}\right)$ ) be the predecessor (resp. successor) of $(x, l)$ in $H$, then the predecessor (resp. successor) of $(a x, l)$ in $H^{\prime}$ will be $\left(a x^{\prime}, l^{\prime}\right)\left(\right.$ resp. $\left.\left(a x^{\prime \prime}, l^{\prime \prime}\right)\right)$.

For $l=0$ and $n$, as $H$ is a 0 -crossing Hamilton cycle, vertices $(x, 0)$ are either of type + or on $H$.

- When $(x, 0)$ is of type + , its predecessor (resp. successor) in the cycle $H$ is $\left(x^{\prime}, n-1\right)$ (resp. $\left(x^{\prime \prime}, 1\right)$ ). Then, in $H^{\prime}$ the predecessor (resp. successor) of $(a x, n)$ will be $\left(a x^{\prime}, n-1\right)$ (resp. $\left(M_{x}(a) x, 0\right)$ ); the predecessor (resp. successor) of $(a x, 0)$ will be $\left(M_{x}^{-1}(a) x, n\right)$ (resp. $\left(a x^{\prime \prime}, 1\right)$ ).

- When $(x, 0)$ is of type -, its predecessor (resp. successor) in $H$ is $\left(x^{\prime}, 1\right)\left(\right.$ resp. $\left(x^{\prime \prime}, n-1\right)$ ). Then, in $H^{\prime}$ the predecessor (resp. successor) of $(a x, 0)$ will be $\left(a x^{\prime}, 1\right)\left(\right.$ resp. $\left.\left(M_{x}^{-1}(a) x, n\right)\right)$; the predecessor (resp. successor) of $(a x, n)$ will be $\left(M_{x}(a) x, 0\right)\left(\right.$ resp. $\left.\left(a x^{\prime \prime}, n-1\right)\right)$ in $H^{\prime}$.

Therefore, when $(x, 0)$ is of type $+($ resp. -$),(a x, n)$ and $(a x, 0)$ are vertices of type $+($ resp. -$)$ in $H^{\prime}$. Hence, all the vertices of levels 0 and $n$ are crossed by $H^{\prime}$; furthermore, the sum of the signs of the vertices of $H^{\prime}$ of levels 0 or $n$ will be $d$ times the sum of the signs of the vertices of $H$ of level 0 , that is, by hypothesis, 0 . Hence, $H^{\prime}$ is 0 -crossing (and also $n$-crossing).

Now, we have to prove that $H^{\prime}$ is effectively a Hamilton cycle. For this it suffices to prove that if we start at some vertex $(a x, 0)$ and follow $H^{\prime}$, we meet successively all the vertices of level 0 and $n$ before coming back to $(a x, 0)$. Indeed, suppose that $(y, l)$ was on the portion of cycle $H$ between $\left(x_{1}, 0\right)$ and $\left(x_{2}, 0\right)$. Then, $(a y, l)$ will be on the portion of $H^{\prime}$ between $\left(a x_{1}, \alpha\right)$ and $\left(a x_{2}, \beta\right)$, where $\alpha=0($ resp. $\alpha=n)$ if $\left(x_{1}, 0\right)$ is of type $+($ resp. -$)$, and $\beta=0($ resp. $\beta=n)$ if $\left(x_{2}, 0\right)$ is of type $($ resp. +). These cases are described on figure (2).

Now, let $\left(x_{0}, 0\right),\left(x_{1}, 0\right), \ldots,\left(x_{d^{n}}=x_{0}, 0\right)$ be the sequence of vertices of $H$ at level 0 in the order we meet them on $H$. Starting from $\left(a_{0} x_{0}, 0\right)$ we will meet successively $\left(a_{1} x_{1}, 0\right),\left(a_{2} x_{2}, 0\right), \ldots$, $\left(a_{d^{n}} x_{d^{n}}=a_{d^{n}} x_{0}, 0\right)$ on $H^{\prime}$. Following such a path, we can meet either $x_{i}$ of type + by going from level $n$ to level 0 , in which case we will apply the perfect matching $M_{x_{i}}$ to some $a$, or $x_{i}$ of type by going from level 0 to $n$, in which case we will apply $M_{x_{i}}^{-1}$ to $a$. So $a_{d^{n}}=\Pi M_{x_{i}}^{\epsilon_{x_{i}}}(a)$ where the product is taken in an order depending on $x_{0}$. As all the $x_{i}$ differ, we can meet again $\left(a_{0} x_{0}, 0\right)$ only at some $a_{q d^{n}} x_{0}$, but $M$ being cyclic-potent, the values $a_{d^{n}}, a_{2 d^{n}}, \ldots, a_{q d^{n}}, \ldots, a_{(d) d^{n}}$ are all distinct. So, we meet again $\left(a_{0} x_{0}, 0\right)$ only after having encountered the $d^{n+1}$ vertices of level 0 .

Now, note that we can perform this construction with $p$ arc-disjoint 0 -crossing cycles and $p$ compatible cyclic-potent families. From construction, the $p 0$-crossing cycles that we will obtain will be arc-disjoint.

Remark 2 When the 0 -crossing Hamilton cycles used in the lemma above are circuits of $\mathcal{W} \overrightarrow{\mathcal{B}} \mathcal{F}(d, n)$, all the vertices are of type + , and the construction leads to circuits of $\mathcal{W B \mathcal { B }} \mathcal{F}(d, n+1)$, giving another proof of the inductive lemma of [5].

RR n 2920 


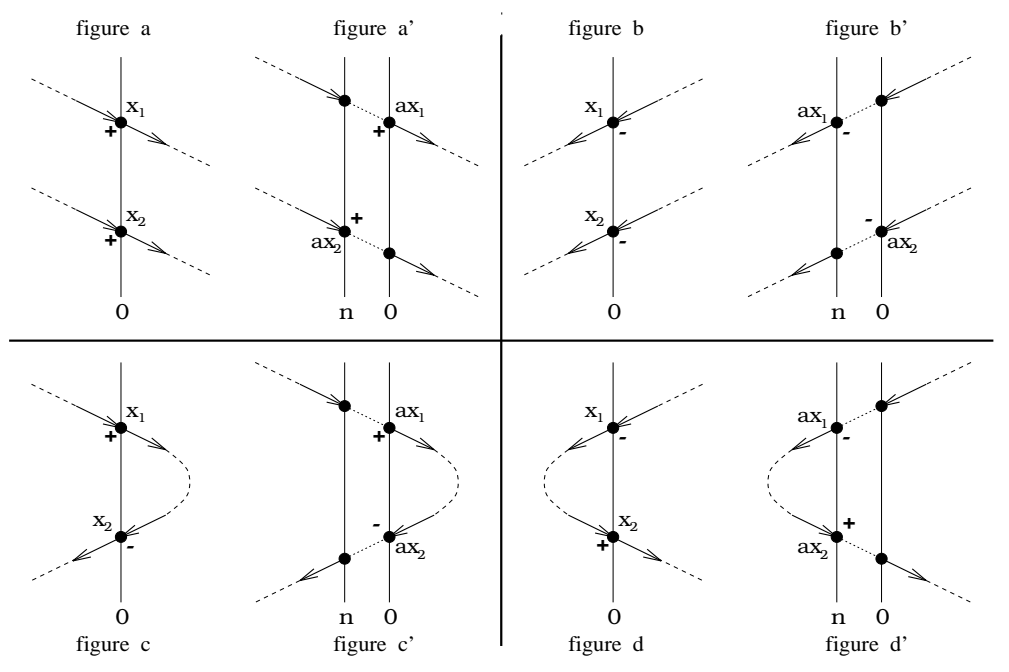

Figure 2: This figure shows the four possible cases when we perform the inductive construction of $\mathcal{W} \overrightarrow{\mathcal{B}} \mathcal{F}(d, n+1)$ from $\mathcal{W} \overrightarrow{\mathcal{B}} \mathcal{F}(d, n)$. In figure $a$ and $a^{\prime}$ (resp. $b$ and $b^{\prime}$ ) the vertices $x_{1}$ and $x_{2}$ are of type $+($ resp. -$)$. Figure $c$ and $c^{\prime}$ (resp. $d$ and $d^{\prime}$ ) displays the case where the vertex $x_{1}$ is of type + (resp. - ) and the vertex $x_{2}$ is of type - (resp. + ).

\section{Decomposition of $\mathcal{W} \overrightarrow{\mathcal{B}} \mathcal{F}(d, n)$}

We will use a decomposition of $\mathcal{W B} \mathcal{B}(d, n)$ into two partial digraphs.

Definition 3.1 The Butterfly digraph $\mathcal{W} \overrightarrow{\mathcal{B}} \mathcal{F}(d, n)$ is the sum of two partial digraphs $\mathcal{W} \overrightarrow{\mathcal{B}} \mathcal{F}_{0,1}(d, n)$ and $\mathcal{W} \overrightarrow{\mathcal{B}} \mathcal{F}_{2, \ldots, d-1}(d, n)$ defined as follows:

- $\mathcal{W} \overrightarrow{\mathcal{B}} \mathcal{F}_{0,1}(d, n)$ contains the arcs which slopes belong to $\{0,1\}$,

- $\mathcal{W} \overrightarrow{\mathcal{B}} \mathcal{F}_{2, \ldots, d-1}(d, n)$ contains the arcs which slopes belong to $\{2, \ldots, d-1\}$.

\subsection{Decomposition of $\mathcal{W} \overrightarrow{\mathcal{B}} \mathcal{F}_{2, \ldots, d-1}(d, n)$}

The proof is inductive on $n$. We start the induction for $n=1$.

Lemma 3.1 When $d \notin\{4,6\}, \mathcal{W B \mathcal { B }} \mathcal{F}_{2, \ldots, d-1}(d, 1)$ is decomposable into Hamilton circuits.

Proof. As $\mathcal{W} \overrightarrow{\mathcal{B}} \mathcal{F}(d, 1)=K_{d}^{+}, \mathcal{W} \overrightarrow{\mathcal{B}} \mathcal{F}_{2, \ldots, d-1}(d, 1)$ is obtained from $K_{d}^{+}$by removing the loops and the arcs of slope 1 . Following Tillson [11], we know that $K_{d}^{+}$without the loops contains $d-1$ arc-disjoint Hamilton circuits when $d \neq 4,6$. So, using Tillson decomposition, we can label the ver- 
tices of $K_{d}^{+}$such that one of the circuits uses all the arcs of slope 1 . By removing it we get $d-2$ arc-disjoint Hamilton circuits in $\mathcal{W} \overrightarrow{\mathcal{B}} \mathcal{F}_{2, \ldots, d-1}(d, 1)$.

Proposition 3.1 For $d \notin\{3,4,6\}, \mathcal{W} \overrightarrow{\mathcal{B}} \mathcal{F}_{2, \ldots, d-1}(d, n)$ is decomposable into Hamilton circuits.

Proof. As $d \notin\{4,6\}$, the proposition is proved for $n=1$ by lemma (3.1). Then, as $d \neq 3$, the $d-2$ compatible cyclic-potent families (3) in section (2.1) use the slopes $\{2, \ldots, d-1\}$ and satisfy the hypothesis of lemma (2.2). Hence, we can apply that lemma inductively, in order to construct $d-2$ arc-disjoint Hamilton circuits (see remark (2)) in $\mathcal{W B}_{\mathcal{B}} \mathcal{F}_{2, \ldots, d-1}(d, n)$.

\subsection{Decomposition of $\mathcal{W} \overrightarrow{\mathcal{B}} \mathcal{F}_{0,1}(d, n)$}

Lemma 3.2 $\mathcal{W B B}_{0,1}(d, 2)$ is decomposable into l-crossing Hamilton cycles.

Proof. For this proof, the vertices of $\mathcal{W} \overrightarrow{\mathcal{B}} \mathcal{F}_{0,1}(d, 2)$ will be denoted by the couples $(x y, l)$ with $x \in \mathbb{Z}_{d}, y \in \mathbb{Z}_{d}$ and $l \in \mathbb{Z}_{2}$. We will show that we can build two arc-disjoint 1-crossing Hamilton cycles in $\mathcal{W} \overrightarrow{\mathcal{B}} \mathcal{F}_{0,1}(d, 2)$ by using two sets of arcs of $\mathcal{W} \overrightarrow{\mathcal{B}} \mathcal{F}_{0,1}(d, 2)$ defined by the next two rules:

1. Arcs of $H_{0}$ :

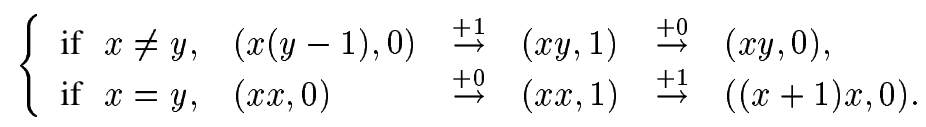

2. Arcs of $H_{1}$ :

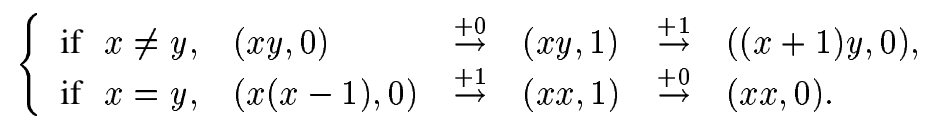

It is easy to verify that $H_{0}$ and $H_{1}$ are arc-disjoint. With the arcs (1) of $H_{0}$, we can define for each $x \in \mathbb{Z}_{d}$ a dipath $P_{x}$ as follows:

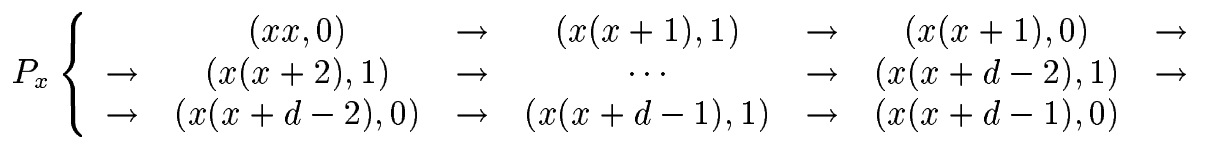

The $d$ dipaths $P_{x}, x \in \mathbb{Z}_{d}$, are clearly vertex-disjoint. Only the vertices noted $(x x, 1)$ are not in these $d$ dipaths. The arcs (2) of $H_{0}$ allows us to join the end vertices of the $d$ dipaths through the missing vertices $(x x, 1)$ as follows: 


$$
\begin{aligned}
P_{x} & \leftarrow((x+d-1)(x+d-1), 1) & \leftarrow P_{x+d-1} & \leftarrow \\
& \leftarrow((x+d-2)(x+d-2), 1) & \leftarrow \cdots & \leftarrow \\
& \leftarrow((x+1)(x+1), 1) & \leftarrow P_{x+1} & \leftarrow \\
& \leftarrow(x x, 1) & \leftarrow P_{x} &
\end{aligned}
$$

One can easily check that we have defined a Hamilton cycle. The $d$ dipaths are joined through their extremal vertices in a cyclic way, using only arcs (2) of $H_{0}$.

By construction, all the vertices at level 1 are crossed. In order to compute the sign of the vertices at level 1 , we can choose to walk along the cycle in the direction $(x x, 0) \rightarrow(x(x+1), 1)$. Therefore, all the vertices $(x y, 1)$ with $x \neq y$ are of type + and have +1 as sign, while the vertices $(x x, 1)$ are of type - and have -1 as sign. So, the sum of the signs is $\left(d^{2}-d\right)-(d) \equiv 0 \quad(\bmod d)$.

To prove that the second set of rules builds a second 1-crossing Hamilton cycle, it suffices to notice that we can rewrite this rule up to a permutation of the letters $x$ and $y$ as being:

- Arcs of $H_{1}$ (with permutation of $x$ and $\left.y\right)$ :

$$
\left\{\begin{array}{lllll}
\text { if } y \neq x, & (y(x+1), 0) & \stackrel{+1}{\leftarrow}(y x, 1) & \stackrel{+0}{\leftarrow}(y x, 0), \\
\text { if } y=x,(y y, 0) & \stackrel{+0}{\leftarrow}(y y, 1) \stackrel{+1}{\leftarrow}((y-1) y, 0) .
\end{array}\right.
$$

Construction 2 is then clearly similar to construction 1 ; to be convinced, just exchange $x$ and $y$, and replace 1 by -1 in the proof for construction (1).

Hence, $H_{0}$ and $H_{1}$ are two arc-disjoint 1-crossing Hamilton cycles. As the levels are equivalent, the result holds also for level 0 .

Figure (3) gives a decomposition of $\mathcal{W} \overrightarrow{\mathcal{B}} \mathcal{F}_{0,1}(3,2)$ into two 1-crossing Hamilton cycles.

Proposition 3.2 For $n \geq 2, \mathcal{W} \overrightarrow{\mathcal{B}} \mathcal{F}_{0,1}(d, n)$ is decomposable into l-crossing Hamilton cycles.

Proof. The proposition is proved for $n=2$ by the lemma (3.2). Then, we use lemma (2.2) with the two compatible cyclic-potent families (2) in section (2.1) which use the slopes $\{0,1\}$ to construct inductively two arcs-disjoint $l$-crossing Hamilton cycles in $\mathcal{W B \mathcal { B }}_{0,1}(d, n)$.

Figure (3) gives the recursive construction of two 1-crossing arc-disjoint Hamilton cycles in $\mathcal{W} \overrightarrow{\mathcal{B}} \mathcal{F}_{0,1}(3,3)$ from two 1 -crossing arc-disjoint cycles in $\mathcal{W B} \mathcal{B}(3,2)$. 

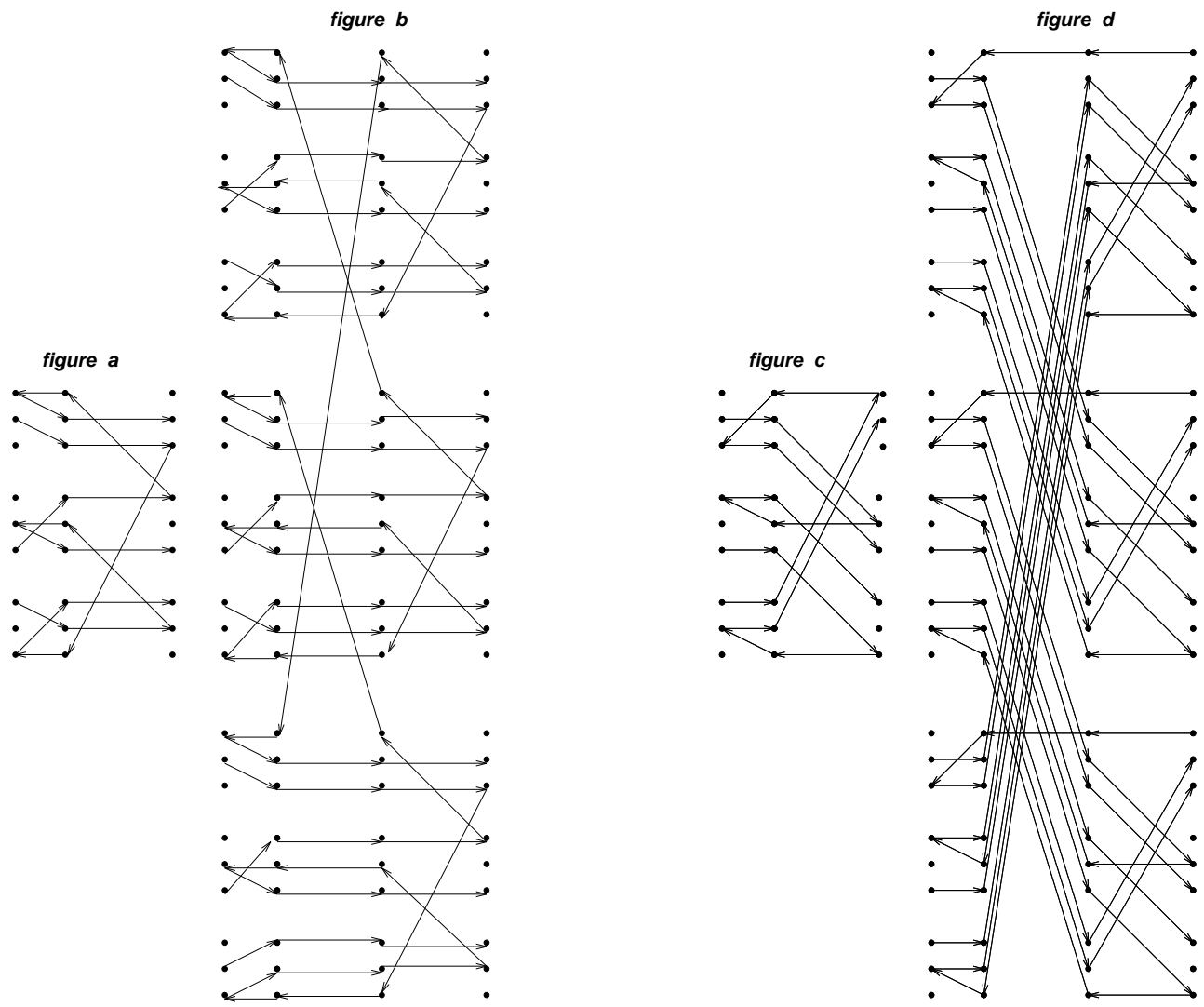

Figure 3: Figures a and c show the two 1 -crossing arc-disjoint Hamilton cycles of $\mathcal{W} \overrightarrow{\mathcal{B}} \mathcal{F}_{0,1}(3,2)$. We display on figures $b$ and d, two 1 -crossing arc-disjoint Hamilton cycles in $\mathcal{W} \overrightarrow{\mathcal{B}} \mathcal{F}_{0,1}(3,3)$ obtained by applying lemma (2.2) with the families (2).

\subsection{Global Decomposition}

We are now ready to prove the main result:

Theorem 3.3 For $n \geq 2$,

- for $d \notin\{3,4,6\}, \mathcal{W B} \mathcal{B}(d, n)$ is decomposable into $d-2$ Hamilton circuits and 2 Hamilton cycles,

- for $d \in\{4,6\}, \mathcal{W B} \mathcal{B}(d, n)$ is decomposable into Hamilton circuits,

- $\mathcal{W} \overrightarrow{\mathcal{B}} \mathcal{F}(3, n)$ is decomposable into 1 Hamilton circuit and 2 Hamilton cycles. 
Proof. According to propositions (3.1) and (3.2) we have, when $d \notin\{3,4,6\}, d-2$ arc-disjoint circuits in $\mathcal{W B B}_{\mathcal{B}_{2, \ldots, d-1}}(d, n)$ and 2 arc-disjoint cycles in $\mathcal{W B B}_{0,1}(d, n)$. So, the result holds in these cases. For $d \in\{4,6\}$ and $n=2$, an exhaustive computer search shows that $\mathcal{W B \mathcal { B }} \mathcal{F}(d, n)$ is decomposable into Hamilton circuits, and so, for $n \geq 2, \mathcal{W B} \mathcal{F}(4, n)$ and $\mathcal{W} \overrightarrow{\mathcal{B}} \mathcal{F}(6, n)$ are decomposable into Hamilton circuits. For $d=3$, we can construct two 1-crossing arc-disjoint Hamilton cycles and one arc-disjoint Hamilton circuit in $\mathcal{W B \mathcal { B }} \mathcal{F}(3,2)$ (see figure (4)). Then, we can apply lemma (2.2) with families (1) and the result holds for $\mathcal{W} \overrightarrow{\mathcal{B}} \mathcal{F}(3, n)$ with $n \geq 2$.

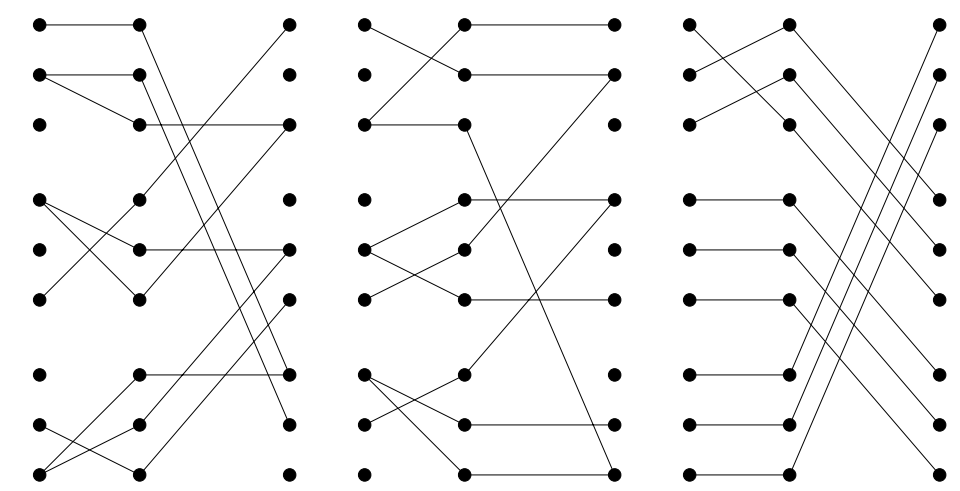

Figure 4: The decomposition of $\mathcal{W} \overrightarrow{\mathcal{B}} \mathcal{F}(3,2)$ into two 1 -crossing arc-disjoint Hamilton cycles and one arc-disjoint Hamilton circuit.

The preceding result implies the conjecture of Barth and Raspaud:

Theorem 3.4 For any $d$ and $n \geq 2, \mathcal{W B} \mathcal{F}(d, n)$ is decomposable into Hamilton cycles.

Remark 3 We could also have derived theorem (3.4) by proving that, if $\mathcal{W B} \mathcal{B}(d, n)$ is decomposable into $l$-crossing Hamilton cycles, then $\mathcal{W B} \mathcal{B}(d, n+1)$ is also decomposable into $l$-crossing Hamilton cycles. This can be done by applying lemma (2.2) with the families (1) in section (2.1). But to start the induction we needed to split the Butterfly digraph into two partial digraphs in order to prove that $\mathcal{W} \overrightarrow{\mathcal{B}} \mathcal{F}(d, 2)$ is decomposable into $l$-crossing Hamilton cycles for $n=2$ and $d \neq 3$.

\section{Conclusion}

In this paper we have proved that $\mathcal{W} \overrightarrow{\mathcal{B}} \mathcal{F}(d, n)$ is always decomposable into Hamilton cycles. However, the problem of decomposing $\mathcal{W B} \mathcal{B}(d, n)$ into Hamilton circuits remains open and is considered in [5]. The difficulty in that case is to start the induction. In fact, we conjecture that $\mathcal{W} \overrightarrow{\mathcal{B}} \mathcal{F}(d, 2)$ is decomposable into Hamilton circuits for $d>3$. Unfortunately such a decomposition is not yet known, even if in [5] we were able to reduce the problem to the case $d$ prime and to solve it in many cases. Consequently, we propose as open problem the following conjecture: 
Conjecture 3 ([5]) For any prime number $p>3, \mathcal{W B \mathcal { B }}(p, 2)$ is decomposable into Hamilton circuits.

Proving this conjecture would completely close the problem of the Hamilton decomposition of the Butterfly network.

\section{Acknowledgements}

We thank the referees for their helpful remarks.

\section{References}

[1] B. Alspach. Research problem 59. Discrete Mathematics, 50:115, 1984.

[2] B. Alspach, J-C. Bermond, and D. Sotteau. Decomposition into cycles I: Hamilton decompositions. In G. Hahn et al., editor, Cycles and Rays, Proceeding Colloquium Montréal, 1987, NATO ASI Ser. C, pages 9-18, Dordrecht, 1990. Kluwer Academic Publishers.

[3] J. Aubert and B. Schneider. Décomposition de la somme cartésienne d'un cycle et de l'union de deux cycles en cycles hamiltoniens. Discrete Mathematics, 38:7-16, 1982.

[4] D. Barth and A. Raspaud. Two edge-disjoint hamiltonian cycles in the Butterfly graph. Information Processing Letters, 51:175-179, 1994.

[5] J-C. Bermond, E. Darrot, O. Delmas, and S. Perennes. Hamilton circuits in directed Butterfly networks. Technical Report RR 95-47, I3S - CNRS URA 1376, 1995. Submitted to Discrete Applied Mathematics.

[6] J-C. Bermond, O. Favaron, and M. Maheo. Hamiltonian decomposition of Cayley graphs of degree 4. Journal of Combinatorial Theory, Series B, 46(2):142-153, 1989.

[7] S.J. Curran and J.A. Gallian. Hamilton cycles and paths in Cayley graphs and digraphs - a survey. To appear in Discrete Mathematics.

[8] F. Thomson Leighton. Introduction to Parallel Algorithms and Architectures: Arrays . Trees . Hypercubes. Computer Science, Mathematics Electrical Engineering. Morgan Kaufmann Publishers, 1992.

[9] Jean de Rumeur. Communication dans les réseaux de processeurs. Collection Etudes et Recherches en Informatique. Masson, 1994. (English version to appear).

[10] D. Sotteau and J. Rowley. Private communication.

[11] T. Tillson. A Hamiltonian decomposition of $K_{2 m}^{*}, 2 m \geq 8$. Journal of Combinatorial Theory, Series B, 29:68-74, 1980.

RR n 2920 
[12] S.A. Wong. Hamilton Cycles and Paths in Butterfly Graphs. Networks, 26(3):145-150, October 1995.

\section{Table of Contents}

1 Introduction and notations 3

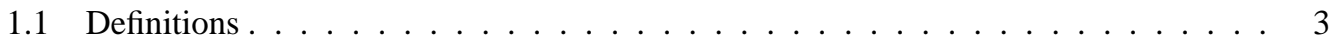

1.2 Results . . . . . . . . . . . . . . . . . . . 5

2 The general construction 6

2.1 Families of perfect matchings . . . . . . . . . . . . . . . 6

2.2 Inductive construction $\ldots \ldots \ldots \ldots \ldots \ldots$

3 Decomposition of $\mathcal{W} \overrightarrow{\mathcal{B}} \mathcal{F}(d, n) \quad 10$

3.1 Decomposition of $\mathcal{W} \overrightarrow{\mathcal{B}} \mathcal{F}_{2, \ldots, d-1}(d, n) \ldots \ldots \ldots \ldots \ldots$

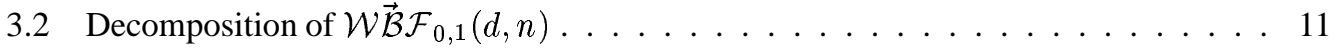

3.3 Global Decomposition . . . . . . . . . . . . . . . . . 13

4 Conclusion $\quad 14$ 


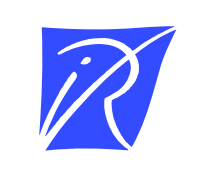

Unité de recherche INRIA Lorraine, Technopôle de Nancy-Brabois, Campus scientifique, 615 rue du Jardin Botanique, BP 101, 54600 VILLERS LÈS NANCY

Unité de recherche INRIA Rennes, Irisa, Campus universitaire de Beaulieu, 35042 RENNES Cedex

Unité de recherche INRIA Rhône-Alpes, 46 avenue Félix Viallet, 38031 GRENOBLE Cedex 1

Unité de recherche INRIA Rocquencourt, Domaine de Voluceau, Rocquencourt, BP 105, 78153 LE CHESNAY Cedex

Unité de recherche INRIA Sophia-Antipolis, 2004 route des Lucioles, BP 93, 06902 SOPHIA-ANTIPOLIS Cedex

Éditeur

INRIA, Domaine de Voluceau, Rocquencourt, BP 105, 78153 LE CHESNAY Cedex (France)

ISSN 0249-6399 